\title{
Validation of a Thin-Layer Chromatography for the Determination of Hydrocortisone Acetate and Lidocaine in a Pharmaceutical Preparation
}

\author{
Małgorzata Dołowy, Katarzyna Kulpińska-Kucia, and Alina Pyka \\ Department of Analytical Chemistry, Faculty of Pharmacy, Medical University of Silesia, PL-4 Jagiellonska Street, \\ 41-200 Sosnowiec, Poland \\ Correspondence should be addressed to Małgorzata Dołowy; mdolowy@sum.edu.pl
}

Received 14 November 2013; Accepted 16 December 2013; Published 6 January 2014

Academic Editors: D. Agbaba and A. M. Rabasco

Copyright (C) 2014 Małgorzata Dołowy et al. This is an open access article distributed under the Creative Commons Attribution License, which permits unrestricted use, distribution, and reproduction in any medium, provided the original work is properly cited.

\begin{abstract}
A new specific, precise, accurate, and robust TLC-densitometry has been developed for the simultaneous determination of hydrocortisone acetate and lidocaine hydrochloride in combined pharmaceutical formulation. The chromatographic analysis was carried out using a mobile phase consisting of chloroform + acetone + ammonia (25\%) in volume composition $8: 2: 0.1$ and silica gel $60 \mathrm{~F}_{254}$ plates. Densitometric detection was performed in UV at wavelengths $200 \mathrm{~nm}$ and $250 \mathrm{~nm}$, respectively, for lidocaine hydrochloride and hydrocortisone acetate. The validation of the proposed method was performed in terms of specificity, linearity, limit of detection (LOD), limit of quantification (LOQ), precision, accuracy, and robustness. The applied TLC procedure is linear in hydrocortisone acetate concentration range of $3.75 \div 12.50 \mu \mathrm{g} \cdot \mathrm{spot}^{-1}$, and from $1.00 \div 2.50 \mu \mathrm{g} \cdot \mathrm{spot}^{-1}$ for lidocaine hydrochloride. The developed method was found to be accurate (the value of the coefficient of variation CV [\%] is less than $3 \%$ ), precise (CV [\%] is less than 2\%), specific, and robust. LOQ of hydrocortisone acetate is $0.198 \mu \mathrm{g} \cdot \mathrm{spot}^{-1}$ and LOD is $0.066 \mu \mathrm{g} \cdot \mathrm{spot}^{-1}$. LOQ and LOD values for lidocaine hydrochloride are 0.270 and $0.090 \mu \mathrm{g} \cdot \mathrm{spot}^{-1}$, respectively. The assay value of both bioactive substances is consistent with the limits recommended by Pharmacopoeia.
\end{abstract}

\section{Introduction}

Corticosteroids are a class of drugs used widely in medicine as an anti-inflammatory and anti-allergic agent. Hydrocortisone acetate is the member of corticosteroid group. This compound usually exists as an ingredient of simple pharmaceutical formulations or in combination with antimicrobial and local anesthetic agents in form of tablets, creams, and injection solutions, respectively. The injection dosage form of hydrocortisone acetate and lidocaine hydrochloride is used to treat many different conditions such as allergic and breathing disorders or skin conditions. Literature concerning quantitative analysis of hydrocortisone acetate in pharmaceutical formulations, especially in form of injection solutions, is relatively sparse. The first report on hydrocortisone acetate analysis in commercial products dated back to 1957, when hydrocortisone acetate in combination with chloramphenicol was simultaneously estimated by UV-spectrophotometric method [1]. In 1967, Furoda and Hashizume proposed Xray diffractometric determination of hydrocortisone acetate in ointment, which consists of hydrocortisone acetate and some antibiotics such as chloramphenicol and tetracycline hydrochloride [2]. A review of current literature shows that among various analytical methods, the modern chromatographic techniques like high performance liquid chromatography in reversed phase system (RP-HPLC) and ultraperformance liquid chromatography (UPLC) are widely applied in analysis of hydrocortisone acetate in various pharmaceutical formulations in form of ointments, creams, and suppositories [3-5]. To our knowledge, only one TLC method has been reported for the estimation of hydrocortisone acetate in combined dosage forms. In 1977, Amin and Jakobs described a direct TLC quantitative analysis of hydrocortisone acetate from ointments and suppositories [6]. 
The methods recommended by Polish and United States Pharmacopoeias for hydrocortisone acetate determination in bulk drug are UV-spectrophotometry and HPLC-UV [7, 8].

Lidocaine hydrochloride (previously known as lignocaine) is local anesthetic amide with antiarrhythmic action. This compound is usually available in tablets and also in form of injectable medicaments used in surgical intervention and in dentistry practice. Literature data on the analysis of lidocaine hydrochloride concerns mostly its determination in biological fluids to control the concentration of lidocaine hydrochloride and its metabolites in human organism for purpose of therapeutic drug monitoring [9]. As it was reported in the literature, lidocaine has been studied in biological samples, for example, in the women plasma after childbirth, in neonatal plasma, in human blood and also in cerebrospinal fluids [9-12]. A thorough literature survey revealed that some high performance liquid chromatography coupled with UV detector and MS/MS spectrometer (LC-UV, LC-MS/MS) and also capillary electrophoresis are available for the estimation of lidocaine hydrochloride in biological fluids [9-11]. Another work indicated that lidocaine and its metabolites in urine samples can be successfully determined by GC-MS method [12]. Lidocaine can exist in simple and combined pharmaceutical formulations (e.g., with cetylpyridinium chloride, cetrimonium bromide, and diclofenac diethylamine or with epinephrine). In pharmaceutical formulations and alginate microspheres containing lidocaine, it has been determined using RP-HPLC with diode array detector (HPLC-DAD) $[13,14]$. Lidocaine is an active substance described in Pharmacopoeia's monograph. According to Polish and United States Pharmacopoeias, the method recommended for lidocaine analysis in pharmaceutical formulations and in bulk drugs is liquid chromatography with UV detection and also potentiometric and acidimetric titration $[7,8]$.

An extensive literature review shows that there are only few reports concerning stability indicating method for simultaneous determination of both pharmacological active compounds: hydrocortisone acetate and lidocaine hydrochloride in combined dosage forms. Out of various analytical methods, the multivariate regression spectrophotometry, micellar capillary electrophoresis (MEKC), and also RP-HPLC coupled with electrochemical detector to quantitation of these substances in combined dosage forms were applied. However, all of those studies have focused almost on the hydrocortisone acetate and lidocaine hydrochloride analysis in form of suppositories and ointments [15-17]. Therefore, attempts were made in this work to develop a fast, sensitive, robust, and cost-effective TLC-densitometric method suitable for quantitative analysis of hydrocortisone acetate and lidocaine hydrochloride in the commercial (injection) dosage form.

This paper is continuation of our previous studies concerning validation of thin-layer chromatography combined with densitometry (TLC-densitometry) for the determination of different bioactive substances in simple and complex dosage forms of many pharmaceutical formulations. In our earlier works, we suggested that the proposed TLCdensitometry may be an alternative method to the modern high performance liquid chromatography in the quality control of above-mentioned substances and it can be applied when HPLC or GC is not affordable in laboratory [18-25]. Additionally, we have stated that the full validation procedure of developed TLC-densitometric method for determination of bioactive substances in pharmaceutical formulations according to validation guidelines [26-29] is necessary for ensuring the reliability of obtained results in comparison to data described by manufacturer.

The objective of this study was to develop the chromatographic conditions suitable for separation and simultaneous determination of hydrocortisone acetate and lidocaine hydrochloride in the presence of substances related to hydrocortisone acetate (hydrocortisone, prednisolone, and cortisone acetate) in combined dosage injection solution (market available) by the use of TLC-densitometry. Hence, the developed TLC-densitometric method was fully evaluated with regard to obligatory validation procedures designed for quality and quantity control of pharmaceutical preparations [26-29].

\section{Materials and Methods}

2.1. Chemicals. The following solvents: ethanol (96\%), chloroform, ammonia (25\%), toluene, and ethyl acetate were purchased from POCh (Gliwice, Poland). Acetone was procured from Chempur (Piekary Śląskie, Poland). All solvents used for the TLC-densitometric analysis were of analytical grade. Hydrocortisone acetate (Ph. Eur. Grade) produced by Fluka Chemicals (Milwaukee, USA), lidocaine hydrochloride (>99\%, Sigma-Aldrich, St. Louis, MO, USA), and the substances related to hydrocortisone acetate such as prednisolone (>99\%, Sigma-Aldrich, St. Louis, MO, USA), cortisone acetate (>99\%, Sigma-Aldrich, St. Louis, MO, USA), and also hydrocortisone (>99\%, Sigma-Aldrich, St. Louis, MO, USA) were used as reference substances in the preparation of standard solutions.

2.2. Preparation of Combine Standard Solution. The standard stock solutions containing hydrocortisone acetate $(125 \mathrm{mg})$ and lidocaine hydrochloride (25 $\mathrm{mg}$ ) were prepared in a volumetric flask by dissolving both reference standard substances in a mixture of acetone + ethanol $(1: 1, \mathrm{v} / \mathrm{v})$ and diluted up to volume $50 \mathrm{~mL}$ with solvent mixture. Final concentration of obtained standard solution was $12.50 \mathrm{mg}$ of hydrocortisone acetate per $5 \mathrm{~mL}$ and $2.50 \mathrm{mg}$ of lidocaine hydrochloride per $5 \mathrm{~mL}$. Next, nine working solutions were prepared by appropriate dilutions of prepared standard solution with the same solvent mixture in order to obtain a concentration of $10.00,8.70,7.50,6.25,5.00,3.75,2.50,1.88$, and $1.25 \mathrm{mg}$ of hydrocortisone acetate in $5 \mathrm{~mL}$ and the $2.00,1.75,1.50,1.25$, $1.00,0.75,0.50,0.38$, and $0.25 \mathrm{mg}$ of lidocaine hydrochloride, respectively, in $5 \mathrm{~mL}$ of solution.

2.3. Pharmaceutical Preparation. The commercial pharmaceutical preparation of the combination, $125 \mathrm{mg}$ of hydrocortisone acetate and $25 \mathrm{mg}$ lidocaine hydrochloride in $5 \mathrm{~mL}$ ampoule, was used. This market available product is used mainly for intraarticular injection. Additionally, each 
ampoule contained respective preservatives as antimicrobial agents.

2.4. Sample Preparation. An ampoule $(5 \mathrm{~mL})$ of the examined product was transferred directly to $50 \mathrm{~mL}$ volumetric flask, dissolved in acetone + ethanol mixture $(1: 1, \mathrm{v} / \mathrm{v})$, and diluted up to the mark with the same solvents. By this way, a sample solution containing $12.50 \mathrm{mg}$ of hydrocortisone acetate and $2.50 \mathrm{mg}$ lidocaine hydrochloride in $5 \mathrm{~mL}$ was obtained. Next, by the use of appropriate dilutions of prepared sample solution, three solutions at concentration of hydrocortisone acetate $10.00,7.50$, and $5.00 \mathrm{mg}$ in $5 \mathrm{~mL}$ and lidocaine hydrochloride $2.00,1.50$, and $1.00 \mathrm{mg}$ in $5 \mathrm{~mL}$ of sample solution were obtained. $5 \mu \mathrm{L}$ of sample solution for the TLC-densitometric analysis and quantitative determination of examined substances, hydrocortisone acetate and lidocaine hydrochloride, was used in each case.

2.5. Apparatus and Chromatographic Conditions. A thinlayer chromatography in normal phase system (NP-TLC) was performed on $10 \mathrm{~cm} \times 20 \mathrm{~cm}$ aluminum plates precoated with silica gel $60 \mathrm{~F}_{254}$ from Merck KGaA (number 1.05570, Darmstadt, Germany). Before using, the plates were activated at $120^{\circ} \mathrm{C}$ for 30 minutes. Next, $5 \mu \mathrm{L}$ of sample was spotted by means of a Camag (Muttenz, Switzerland) micropipette $(5 \mu \mathrm{L})$. The plates were developed at room temperature $\left(20^{\circ} \mathrm{C}\right)$ in horizontal chamber from Camag $20 \mathrm{~cm} \times 20 \mathrm{~cm}$ (number 0.222 .5255 , Muttenz, Switzerland) with the use of chloroform + acetone + ammonia (25\%) in volume composition $8: 2: 0.1$. The chamber was previously saturated with vapors of mobile phase for 30 minutes. The developing distance was $7.5 \mathrm{~cm}$. Finally, the plates were dried for $20 \mathrm{~h}$ at $20^{\circ} \mathrm{C}$ in a fume cupboard. Additionally, a twin-trough chamber produced by Camag $20 \mathrm{~cm} \times 10 \mathrm{~cm}$ (number 0.222 .5221 , Muttenz, Switzerland) and silica gel $60 \mathrm{~F}_{254}$ (number 1.05554, Darmstadt, Germany) was used to robust test. Densitometric and spectrodensitometric analysis was carried out by densitometer (Camag, Muttenz, Switzerland) operated in absorbance mode and equipped with TLC Scanner 3. All the parameters were controlled by WinCATS 1.4.2 software. Deuterium lamp was used as a source of radiation. After spectrodensitometric analysis, densitometric determination of hydrocortisone acetate was made at absorption maximum equal to $250 \mathrm{~nm}$ and for lidocaine hydrochloride at a wavelength $200 \mathrm{~nm}$.

2.6. Method Validation. The proposed method was validated in terms of range, specificity, linearity, precision, accuracy, quantification limit (LOQ), detection limit (LOD), and robustness in accordance with established International Conference on Harmonization (ICH) guideline [26] and validation guides published by Ferenczi-Fodor et al. [27, 28] and also by Nagy-Turák et al. [29].

2.6.1. Specificity. Specificity of the method was checked by TLC-chromatography of working standard solutions of hydrocortisone acetate, lidocaine hydrochloride, and substances related to hydrocortisone acetate (prednisolone, hydrocortisone, and cortisone acetate) and sample solution containing hydrocortisone acetate and lidocaine hydrochloride obtained from the commercial product. In order to determine the specificity, appropriate chromatographic conditions were applied (such as suitable adsorbent and a proper mobile phase), which allowed the complete separation of hydrocortisone acetate and lidocaine hydrochloride in the presence of substances related to hydrocortisone acetate, mentioned above. To estimate the specificity of developed method, comparison of obtained chromatographic bands was made.

Moreover, the effect of resolution of both examined bioactive substances and the other ones presented in the examined sample, including the substances related to hydrocortisone acetate and preservatives, the following separation factor for active constituents was calculated [30]:

$$
R_{S}=\frac{2 d}{w_{b 1}+w_{b 2}},
$$

where $d$ is distance between the centers of two adjacent chromatographic bands $w_{b 1}$ and $w_{b 2}$ that are band width at base.

2.6.2. Linearity. Linearity test of developed TLCdensitometric method was made by the dilution of the reference standard solutions of hydrocortisone acetate and lidocaine hydrochloride to the required concentration. For this aim, a series of nine solutions containing 10.00, 8.70, $7.50,6.25,5.00,3.75,2.50,1.88$, and $1.25 \mathrm{mg}$ of hydrocortisone acetate in $5 \mathrm{~mL}$ of solution and $2.00,1.75,1.50,1.25,1.00$, $0.75,0.50,0.38$, and $0.25 \mathrm{mg}$ of lidocaine hydrochloride in $5 \mathrm{~mL}$ of solution were prepared. Five $\mu \mathrm{L}$ of each solution was spotted on previously activated TLC plate. The plates were developed using a mobile phase consisting of chloroform + acetone + ammonia (25\%) in volume composition $8: 2: 0.1$ and next scanned. This analysis was repeated three times. Calibration curve was plotted between peak area $A$ [AU] versus concentration of examined substances $x\left[\mu \mathrm{g} \cdot \mathrm{spot}^{-1}\right]$. The coefficient correlation, slope, and intercept of obtained calibration plots were reported.

2.6.3. Limit of Detection (LOD) and Quantification (LOQ). The LOD and LOQ values were determined on the basis of specific calibration curve using the samples containing both reference standards at respective concentrations:

(i) $0.50,0.40$, and $0.30 \mathrm{mg}$ of hydrocortisone acetate in $5 \mathrm{~mL}$ of solution,

(ii) $0.75,0.50$, and $0.38 \mathrm{mg}$ of lidocaine hydrochloride in $5 \mathrm{~mL}$ of solution.

Five $\mu \mathrm{L}$ of prepared solutions was used in this analysis. The results are the means of three measurements.

The detection limit (LOD) was calculated as

$$
\mathrm{LOD}=\frac{3.3 \sigma}{S} .
$$

The quantification limit (LOQ) was calculated as

$$
\mathrm{LOQ}=\frac{10 \sigma}{S},
$$


TABLE 1: Experimental design matrix $\left(2^{3}\right)$ for robustness test for biological active substances coming from injection dosage form of examined drug.

\begin{tabular}{|c|c|c|c|c|c|c|c|c|c|}
\hline \multirow[t]{2}{*}{ Experiment no. } & \multirow[t]{2}{*}{$X_{1}$} & \multirow[t]{2}{*}{$X_{2}$} & \multirow[t]{2}{*}{$X_{3}$} & \multirow[t]{2}{*}{$X_{4}$} & \multirow[t]{2}{*}{$X_{5}$} & \multirow[t]{2}{*}{$X_{6}$} & \multirow[t]{2}{*}{$X_{7}$} & \multicolumn{2}{|c|}{$\begin{array}{l}\text { Biological active substance }{ }^{\mathrm{a}} \text { content } \\
\left(y_{i}\right)[\mathrm{mg}] \text { in examined drug }\end{array}$} \\
\hline & & & & & & & & HA & $\mathrm{L}$ \\
\hline 1 & + & + & + & + & + & + & + & 123.7 & 24.8 \\
\hline 2 & + & + & - & + & - & - & - & 119.5 & 25.9 \\
\hline 3 & + & - & + & - & - & + & - & 124.3 & 25.6 \\
\hline 4 & + & - & - & - & + & - & + & 121.4 & 24.4 \\
\hline 5 & - & + & + & - & + & - & - & 126.0 & 24.2 \\
\hline 6 & - & + & - & - & - & + & + & 123.9 & 24.5 \\
\hline 7 & - & - & + & + & - & - & + & 120.1 & 24.6 \\
\hline 8 & - & - & - & + & + & + & - & 120.8 & 25.6 \\
\hline \multicolumn{10}{|c|}{$\begin{array}{l}\text { Effect for HA, L in examined } \\
\text { drug }\end{array}$} \\
\hline HA & -0.475 & 1.625 & 2.125 & -2.875 & 1.025 & 1.425 & -0.375 & & \\
\hline $\mathrm{L}$ & 0.450 & -0.200 & -0.300 & 0.550 & -0.400 & 0.350 & -0.750 & & \\
\hline
\end{tabular}

${ }^{\mathrm{a}} \mathrm{HA}$ : hydrocortisone acetate; L: lidocaine hydrochloride.

$X_{1}$ : sorbent type; $X_{2}$ : chamber type; $X_{3}$ : temperature of plate activation; $X_{4}$ : distance of development; $X_{5}$ : saturation time of the chamber; $X_{6}$ : volume of chloroform; $X_{7}$ : volume of acetone.

where $\sigma=$ the standard deviation of the response and $S=$ the slope of the calibration curve.

2.6.4. Accuracy. The accuracy of the method was evaluated by measurement of recovery. Percent recovery was performed by the standard addition method. For this aim, known amounts of reference standard hydrocortisone acetate and lidocaine hydrochloride were added to the sample in given quantity: $50 \%, 100 \%$, and $150 \%$ level of test concentration $(10.00,7.50$, and 5.00 per $5 \mathrm{~mL}$ for hydrocortisone acetate) and $(2.00,1.50$, and 1.00 per $5 \mathrm{~mL}$ for lidocaine hydrochloride). This analysis was performed six times. The percentage of recovery for both drug components was calculated.

2.6.5. Precision. Repeatability (intraday precision) of the method was determined by the analysis of three replicates of sample solutions at three different concentrations of hydrocortisone acetate $(10.00,7.50$, and 5.00 per $5 \mathrm{~mL}$ of solution) and for lidocaine hydrochloride $(2.00,1.50$, and 1.00 per $5 \mathrm{~mL}$ of solution). All solutions were prepared independently and repeated three times. $5 \mu \mathrm{L}$ of prepared solutions was used in each case. Precision was determined on the basis of densitometric measurements of obtained spots as the relative standard deviation (coefficient of variation: CV [\%]).

2.6.6. Robustness. The robustness of the method was evaluated during its development by making changes to the method parameters. Robustness test was prepared according to guidelines described in the papers by FerencziFodor et al. [27, 28] and Nagy-Turák et al. [29]. 7.50 and $1.50 \mu \mathrm{g} \cdot \mathrm{spot}^{-1}$ of hydrocortisone acetate and lidocaine hydrochloride, respectively, were spotted on the plates and next the plates were developed after altering the conditions. The conditions changed were the sorbent type (number
1.05570 , number 1.05554$)$, the chamber type $(20 \mathrm{~cm} \times 10 \mathrm{~cm}$, $20 \mathrm{~cm} \times 20 \mathrm{~cm})$, the temperature of plate activation $\left( \pm 10^{\circ} \mathrm{C}\right)$, the distance of development $( \pm 0.5 \mathrm{~cm})$, saturation time of the chamber $( \pm 2 \mathrm{~min})$, the volume of chloroform $( \pm 0.1 \mathrm{~mL})$, and the volume of acetone $( \pm 0.1 \mathrm{~mL})$ in used mobile phase. The main effects of seven factors were tested on two levels in eight experiments [27-29]. The levels of factors investigated and the experimental design matrix $\left(2^{3}\right)$ are shown in Table 1. A high level is represented by "+" and a low level by "-." The ways of calculation of the effects $(E)$ characterizing the particular individual factors and rank probabilities were earlier presented [18-23].

\section{Results and Discussion}

3.1. Optimization of TLC Method. In our previous paper [19], we confirmed that normal phase thin-layer chromatography (NP-TLC) combined with densitometry is a method which can be successfully applied for identification and quantification of hydrocortisone in a simple pharmaceutical formulation in form of tablets containing $20 \mathrm{mg}$ of hydrocortisone per tablet. We found that the optimal separation of hydrocortisone from its related substances: hydrocortisone acetate and prednisolone can be achieved on silica gel $60 \mathrm{~F}_{254}$ and by the use of mobile phase: acetone $+n$-hexane + glacial acetic acid in volume composition $5 \mathrm{~mL}$ : $5 \mathrm{~mL}$ : $0.05 \mathrm{~mL}$. Based on these results, elaboration of the TLCdensitometric method for quantitation of hydrocortisone acetate in combined with lidocaine hydrochloride injection solution (ampoule $5 \mathrm{~mL}$ ) was begun from optimization of the chromatographic conditions, necessary for simultaneous determination of hydrocortisone acetate and lidocaine hydrochloride in this solution. Therefore, besides the above mentioned mixture, the new two mobile phases were applied 
to develop the chromatographic plates for analysis of sample solution and the reference standard solutions of drug components. The first mobile phase used in experiment was a mixture of the following solvents: chloroform + acetone + ammonia (25\%) in volume composition $8: 2: 0.1$ and the second mobile phase used was a mixture of toluene + ethyl acetate in volume composition $15: 35$. Finally, the obtained results indicated that the optimum mobile phase for separation of lidocaine hydrochloride, hydrocortisone acetate, and also the substances related to hydrocortisone acetate, for example, hydrocortisone, prednisolone, and cortisone acetate, is the first mobile phase. Application of chloroform + acetone + ammonia (25\%) as a mobile phase resulted in obtaining compact bands and symmetric peaks for both examined substances. Additionally, a confirmation of the presence of preservatives in the sample of studied drug was possible. Thus, it can be suggested that the optimized NPTLC technique is suitable for quantitative determination of hydrocortisone acetate and lidocaine hydrochloride in combined preparations in form of injectable solution.

3.1.1. Method Validation. Summarized results of the method validation are presented in Figures $1-5$ as well as in Tables 1 and 2.

3.1.2. Specificity. In order to verify the specificity of applied TLC-densitometric method, the mixture of chloroform + acetone + ammonia (25\%) in volume composition $8: 2: 0.1$ was used. As we described above, this mixture was chosen for development as the optimum mobile, because it enabled a successful separation of hydrocortisone acetate (HA) from lidocaine hydrochloride (L) and from its related substances, namely, prednisolone (PR), cortisone acetate (CRA), and hydrocortisone $(\mathrm{H})$ presented in commercial injection solution. Typical densitogram obtained from combine standard solution under these chromatographic conditions is performed in Figure 1(a).

On the basis of Figure 1(a), it can be observed that the hydrocortisone $(\mathrm{H})$ and prednisolone $(\mathrm{PR})$ form one compact band with $R_{F}$ value $0.07 \pm 0.01$. The $R_{F}$ values of hydrocortisone acetate (HA), cortisone acetate (CRA), and lidocaine hydrochloride (L) are as follows: $R_{F(\mathrm{HA})}=0.41 \pm$ $0.02, R_{F(\mathrm{CRA})}=0.55 \pm 0.02$, and $R_{F(\mathrm{~L})}=0.85 \pm 0.03$. While the rate of $R_{S}$ (calculated accordance to (1)) received the following: $R_{S(\mathrm{H}, \mathrm{PR} / \mathrm{HA})}=3.30, R_{S(\mathrm{HA} / \mathrm{CRA})}=1.17$, and $R_{S(\mathrm{CRA} / \mathrm{L})}$ $=3.43$. Densitometric scan of obtained bands was made at the maximum absorption $\left(\lambda_{\max }\right)$ for each substance. In the case of hydrocortisone, prednisolone, and hydrocortisone acetate, the wavelength $250 \mathrm{~nm}$ was used, for cortisone acetate $247 \mathrm{~nm}$, and for lidocaine hydrochloride $200 \mathrm{~nm}$. The high selectivity of the adsorption TLC combined with densitometry for the quantification of hydrocortisone acetate and lidocaine hydrochloride in the pharmaceutical preparation in form of injection solution confirms densitogram of lidocaine hydrochloride (L) and hydrocortisone acetate (HA) obtained from examined drug, which was shown in Figure 1(b). No interference from substances related to hydrocortisone acetate and other components (e.g., preservatives) and contamination was observed. Moreover, additional components like preservatives (CS) present in the examined drug were well separated from hydrocortisone acetate and lidocaine hydrochloride, and it did not affect the TLCdensitometric analysis of HA and L. The value of $R_{F(\mathrm{CS})}$ is $0.64 \pm 0.02$, while the main band of CS occurs at $200 \mathrm{~nm}$. The average $R_{F}$ values of hydrocortisone acetate and lidocaine hydrochloride were 0.41 and 0.84 , respectively. The $R_{F}$ values of hydrocortisone acetate and lidocaine hydrochloride, which were determined in the examined drug, are compatible with the $R_{F}$ values of reference standard hydrocortisone acetate and lidocaine hydrochloride. Moreover, the specificity of developed method confirms the spectrodensitograms of examined substances: lidocaine hydrochloride and hydrocortisone acetate obtained from commercial product, which are identical with spectrodensitograms of reference standards of lidocaine hydrochloride and hydrocortisone acetate.

3.1.3. Accuracy. Accuracy of the TLC-densitometric method was evaluated by the measurement of recovery. Recovery of hydrocortisone acetate in the examined drug ranged from $95.4 \%$ to $101.4 \%$, from $97.1 \%$ to $101.4 \%$, and from $95.1 \%$ to $101.4 \%$, respectively, for $50 \%, 100 \%$, and $150 \%$ amount of standard hydrocortisone acetate added to the pharmaceutical preparation. Generally, the average recovery was equal to $97.6 \%, 99.0 \%$, and $98.0 \%$. Recovery of lidocaine hydrochloride coming from the same pharmaceutical preparation (injection solution) ranged from $95.3 \%$ to $100.3 \%$, from $95.3 \%$ to $98.7 \%$, and from $97.2 \%$ to $101.6 \%$ for, respectively, $50 \%$, $100 \%$, and $150 \%$ standard lidocaine hydrochloride added to a solution of pharmaceutical formulation. An average recovery was equal to $97.6 \%, 97.0 \%$, and $99.3 \%$ (Table 2). The values of the coefficient of variation less than $3 \%$ confirm the accuracy of the proposed method for the quantitative determination of hydrocortisone acetate and lidocaine hydrochloride in the combined pharmaceutical preparation.

3.1.4. Linearity. Linearity of the proposed TLC method was determined by plotting peak area $(A)[\mathrm{AU}]$ versus concentration $(x)$ of the proper standard substance: hydrocortisone acetate or lidocaine hydrochloride respectively, in $\mu \mathrm{g} \cdot \mathrm{spot}^{-1}$ (Table 2). The plot $(n=7)$ was linear for hydrocortisone acetate in the range from $3.75 \mu \mathrm{g} \cdot \mathrm{spot}^{-1}$ to $12.50 \mu \mathrm{g} \cdot \mathrm{spot}^{-1}$ (Figure 2(a)). The calibration curve $(n=6)$ for lidocaine hydrochloride was linear in the range from $1.00 \mu \mathrm{g} \cdot \mathrm{spot}^{-1}$ to $2.50 \mu \mathrm{g} \cdot \mathrm{spot}^{-1}$, as it is shown in Figure 3(a). For both linear relationships, the high values of the correlation coefficients were achieved $(r>0.999)$. The statistical data of obtained linear dependencies, which confirm excellent linearity of two calibration curves, are presented in Table 2. Moreover, in both cases, the plots of residuals against the concentration of hydrocortisone acetate or lidocaine hydrochloride, respectively, were prepared. Difference between the obtained values and those estimated using a proper calibration curve is small. The residuals were distributed above and below the zero residual line for both examined substances (Figures 2(b) and $3(b))$. 


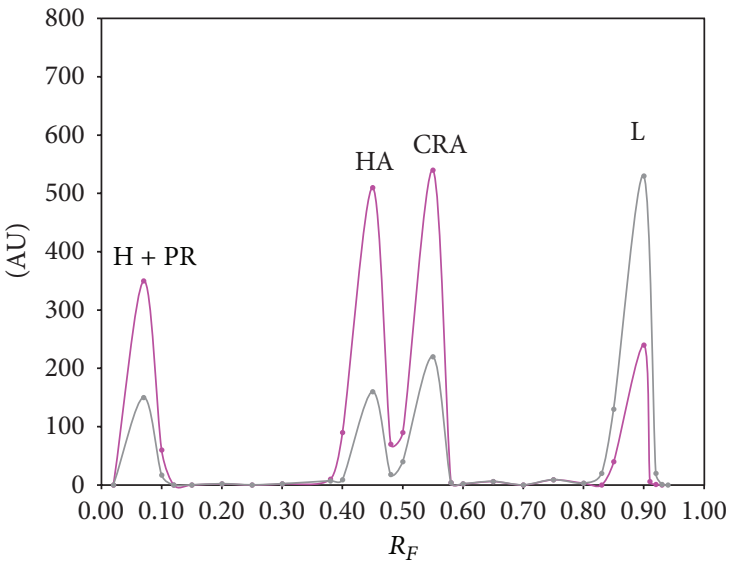

(a)

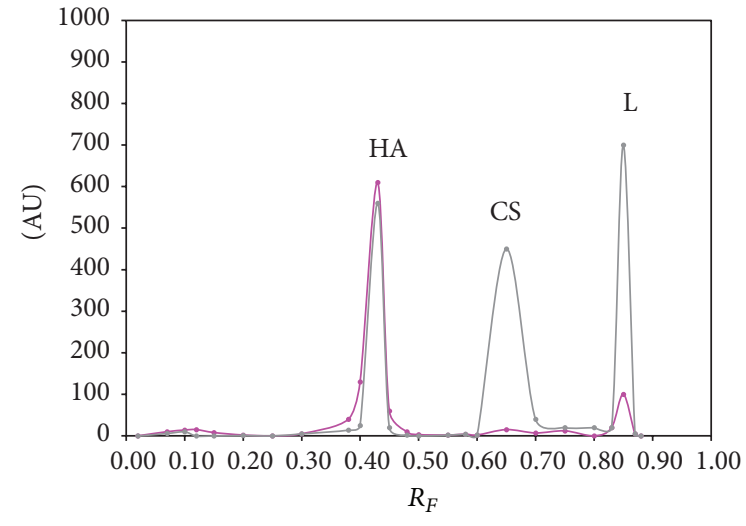

(b)

FIgURE 1: (a) Densitogram of the combine standard solution containing the following reference substances: lidocaine hydrochloride (L), hydrocortisone acetate (HA) and its related substances, namely, hydrocortisone (H), prednisolone (PR), and cortisone acetate (CRA) and (b) densitogram of drug sample analyzed by NP-TLC, at wavelengths of $200 \mathrm{~nm}$ and $250 \mathrm{~nm}$ obtained under optimum chromatographic conditions.

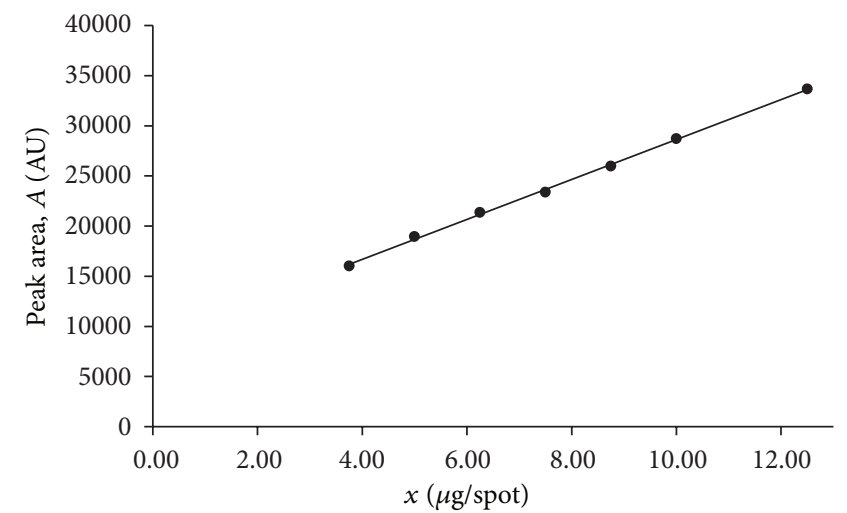

(a)

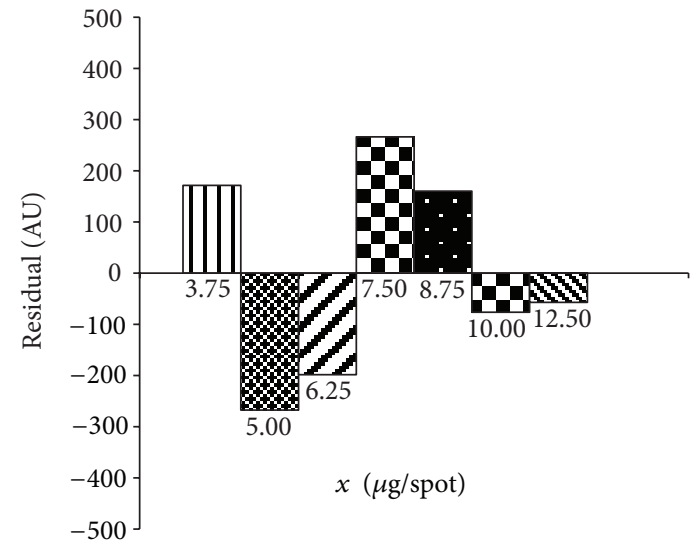

(b)

FIGURE 2: Calibration plot for hydrocortisone acetate (a) and plot of residuals (b) for hydrocortisone acetate in linear working range.

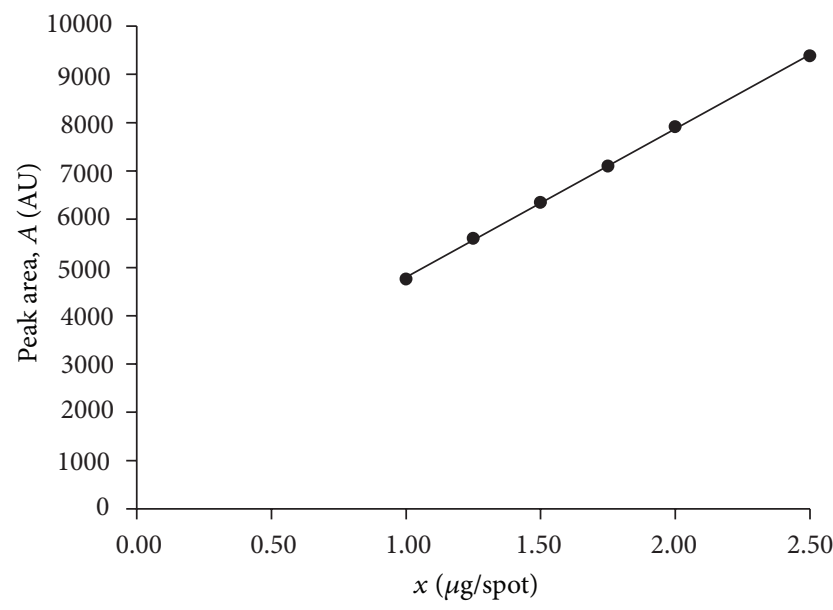

(a)

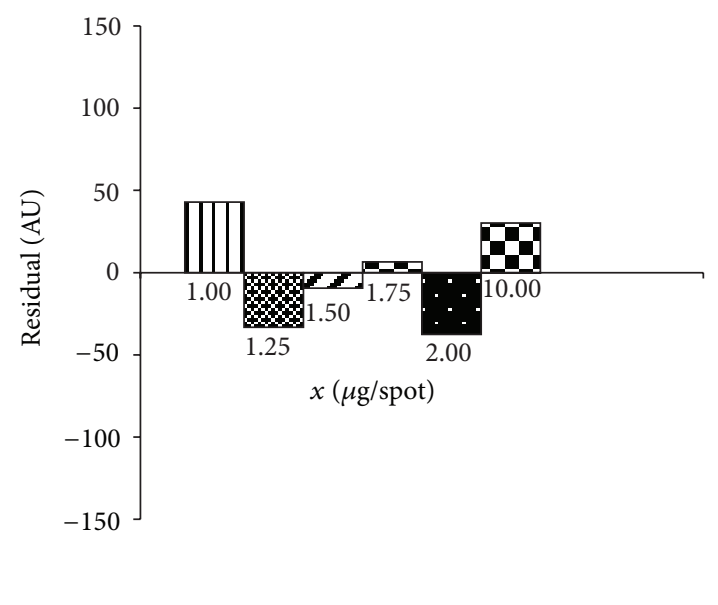

(b)

FIgURE 3: Calibration plot for lidocaine hydrochloride (a) and plot of residuals (b) for lidocaine hydrochloride in linear working range. 


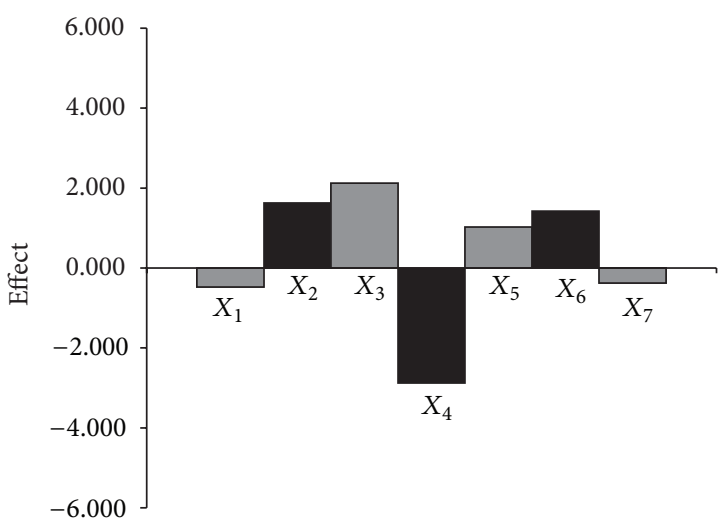

(a)

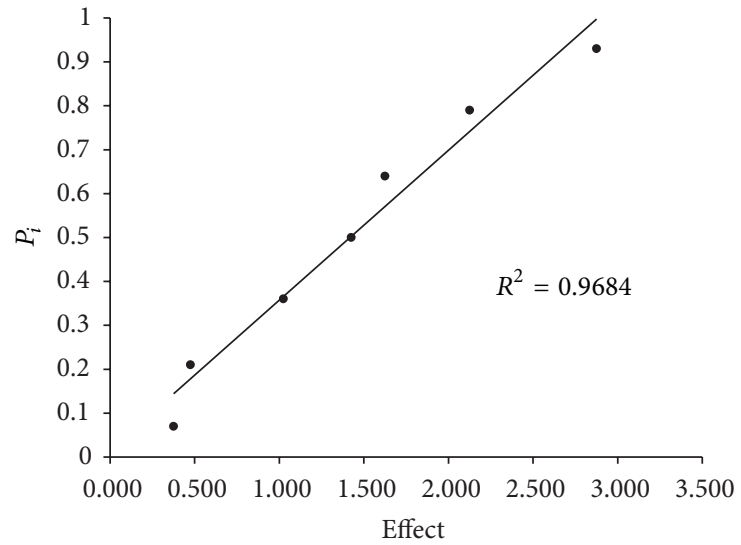

(b)

FIGURE 4: Robustness test: the effect of the factors (a) and a half-normal probability plot of effects (b) for determination of hydrocortisone acetate in examined drug.

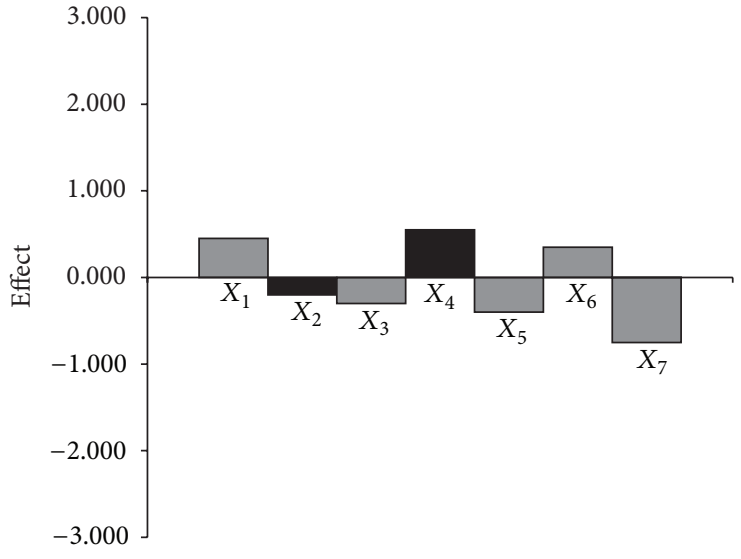

(a)

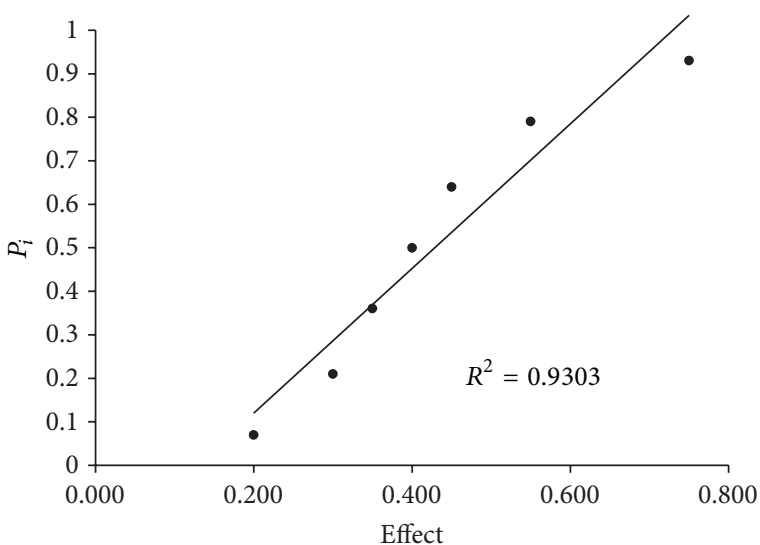

(b)

FIGURE 5: Robustness test: the effect of the factors (a) and half-normal probability plot of effects (b) for determination of lidocaine hydrochloride in examined drug.

3.1.5. Precision. Precision of the proposed method was investigated by densitometric measurements of spots obtained on the basis of sample solutions prepared at three different concentrations of hydrocortisone acetate and lidocaine hydrochloride (listed in Section 2). The results of this experiment were expressed in CV [\%] (coefficient of variation) presented in Table 2. The results of CV for hydrocortisone acetate and lidocaine hydrochloride in the examined drug ranged from $0.92 \%$ to $1.32 \%$ and from $0.97 \%$ to $1.69 \%$, respectively. The values of the coefficients of variation were less than $2 \%$ in each case. This fact indicates the closeness of agreement between a series of measurements obtained and confirms precision of developed TLC-densitometric method.

3.1.6. Quantification Limit (LOQ) and Detection Limit (LOD). As it was described previously, the limits of detection and quantification of the developed method were determined on the basis of calibration plot. The limits of quantification and detection for hydrocortisone acetate are, respectively,
$\mathrm{LOQ}=0.198 \mu \mathrm{g} \cdot \mathrm{spot}^{-1}$ and LOD $=0.066 \mu \mathrm{g} \cdot \mathrm{spot}^{-1}$. The limits of quantification and detection for lidocaine hydrochloride are, respectively, LOQ $=0.270 \mu \mathrm{g} \cdot \operatorname{spot}^{-1}$ and LOD $=$ $0.090 \mu \mathrm{g} \cdot \mathrm{spot}^{-1}$.

The values obtained for LOD and LOQ were indicative of high sensitivity of the method for both substances presented in examined injection solution.

3.1.7. Robustness. As we previously described, the robustness of the TLC-densitometric procedure was evaluated during development by making small, but deliberate, changes to the method parameters. The main effects of seven factors were tested on two levels in eight experiments. Table 1 shows the results obtained for hydrocortisone acetate and lidocaine hydrochloride content $\left(y_{i}\right)$ in the sample of examined injectable solution. On the basis of these results, it can be concluded that no factor has significant effect on the results. Moreover, the results were also evaluated by half-normal probability plot of rank probabilities $\left(p_{i}\right)$ as a function of 
TABLE 2: Validation of the method. Data for the quantitative determination of hydrocortisone acetate and lidocaine hydrochloride by NP-TLC with densitometry ${ }^{\mathrm{a}}$.

\begin{tabular}{|c|c|c|}
\hline Method characteristic & $\begin{array}{l}\text { Results } \\
\text { Hydrocortisone acetate }\end{array}$ & $\begin{array}{l}\text { Results } \\
\text { Lidocaine hydrochloride }\end{array}$ \\
\hline Specificity & Specific & Specific \\
\hline Range $\left[\mu \mathrm{g} \cdot \operatorname{spot}^{-1}\right]$ & $3.75 \div 12.50$ & $1.00 \div 2.50$ \\
\hline Linearity $\left[\mu \mathrm{g} \cdot \mathrm{spot}^{-1}\right]$ & $\begin{array}{c}A=8721.6( \pm 245.4)+1990.6( \pm 30.0) \cdot x \\
n=7 ; r=0.9994 ; s=221.7 ; F=4391.5 \\
P<0.0001\end{array}$ & $\begin{array}{c}A=1722.4( \pm 52.9)+3075.5( \pm 30.4) \cdot x \\
n=6 ; r=0.9998 ; s=36.7 ; F=10223 ; \\
P<0.0001\end{array}$ \\
\hline \multicolumn{3}{|l|}{ Accuracy } \\
\hline for $50 \%$ standard solutions added $(n=6)$ & $R=97.6 \% \mathrm{CV}=2.60 \%$ & $R=97.6 \% \mathrm{CV}=2.12 \%$ \\
\hline for $100 \%$ standard solutions added $(n=6)$ & $R=99.0 \% \mathrm{CV}=1.62 \%$ & $R=97.0 \% \mathrm{CV}=1.44 \%$ \\
\hline for $150 \%$ standard solutions added $(n=6)$ & $R=98.0 \% \mathrm{CV}=2.53 \%$ & $R=99.3 \% \mathrm{CV}=1.85 \%$ \\
\hline $\begin{array}{l}\text { Detection limit (LOD) } \\
{\left[\mu \mathrm{g} \cdot \mathrm{spot}^{-1}\right]}\end{array}$ & 0.066 & 0.090 \\
\hline $\begin{array}{l}\text { Quantification limit (LOQ) } \\
{\left[\mu \mathrm{g} \cdot \mathrm{spot}^{-1}\right]}\end{array}$ & 0.198 & 0.270 \\
\hline \multicolumn{3}{|l|}{ Precision $(C V[\%])$} \\
\hline $\begin{array}{r}\text { for } 10.0 \text { hydrocortisone acetate and } 2.0 \\
\text { lidocaine hydrochloride } \mu \mathrm{g} \cdot \operatorname{spot}^{-1}(n=3)\end{array}$ & $0.93 \%$ & $1.01 \%$ \\
\hline $\begin{array}{l}\text { for } 7.5 \text { hydrocortisone acetate and lidocaine } \\
\text { hydrochloride } 1.5 \mu \mathrm{g} \cdot \operatorname{spot}^{-1}(n=3)\end{array}$ & $0.92 \%$ & $1.69 \%$ \\
\hline $\begin{array}{l}\text { for } 5.0 \text { hydrocortisone acetate and } 1.0 \text { lidocaine } \\
\text { hydrochloride } \mu \mathrm{g} \cdot \operatorname{spot}^{-1}(n=3)\end{array}$ & $1.32 \%$ & $0.97 \%$ \\
\hline Robustness (CV [\%]) & Robust & Robust \\
\hline
\end{tabular}

${ }^{\mathrm{a}} A$ : peak area $[\mathrm{AU}] ; x$ : amount of analyzed drug $\left[\mu \mathrm{g} \cdot \operatorname{spot}^{-1}\right] ; r$ : correlation coefficient; $R$ : recovery [\%]; CV: coefficient of variation [\%].

TABLE 3: Statistical data of the results of quantitative determination of hydrocortisone acetate and lidocaine hydrochloride in injection dosage form of investigated commercial product by NP-TLC with densitometry.

\begin{tabular}{|c|c|c|}
\hline Parameter & $\begin{array}{l}\text { Hydrocortisone } \\
\text { acetate }\end{array}$ & $\begin{array}{c}\text { Lidocaine } \\
\text { hydrochloride }\end{array}$ \\
\hline Number of analyses & 6 & 6 \\
\hline $\begin{array}{l}\text { The declared amount of respective substance in } \\
\text { one ampoule [mg] }\end{array}$ & 125 & 25 \\
\hline The average value of content [mg] & 118.7 & 24.6 \\
\hline Minimum $[\mathrm{mg}]$ & 115.9 & 23.7 \\
\hline Maximum [mg] & 120.9 & 24.9 \\
\hline Variance $\left(s^{2}\right)$ & 3.66 & 0.20 \\
\hline Standard deviation (SD) & 1.9 & 0.4 \\
\hline $\begin{array}{l}\text { Coefficient of variation } \\
(\mathrm{CV}[\%])\end{array}$ & 1.60 & 1.79 \\
\hline $\begin{array}{l}\text { Confidence interval of the mean value at } \\
\text { significance level } 95 \%\end{array}$ & $\mu=118.7 \pm 1.9$ & $\mu=24.6 \pm 0.4$ \\
\hline $\begin{array}{l}\text { Content [\%] of bioactive substance calculated in } \\
\text { relation to the value declared by manufacturer }\end{array}$ & $95.0 \%$ & $98.4 \%$ \\
\hline
\end{tabular}

the absolute values of the main effects. The effects of these factors and half-normal probability plot of the effects for the determination of hydrocortisone acetate and lidocaine hydrochloride in examined product are presented in Figures 4 and 5, respectively. The points of all factors lie near in the straight line, which indicates that their effect is negligible $\left(r^{2} \geq 0.9303\right)$. Thus, the presented TLC-densitometric method was found to be robust with respect to variability in applied chromatographic conditions, except the variation of the ammonia (25\%) content. The content of ammonia should be constant.

3.1.8. Application of the Developed TLC-Densitometric Method to Pharmaceutical Formulation. The commercial injection solution of the hydrocortisone acetate and lidocaine 
hydrochloride mixture was successfully analyzed by the use of proposed TLC-method. The results of densitometric analysis of hydrocortisone acetate in form of densitometric bands coming from sample in which declared amount is equal to $7.50 \mu \mathrm{g} \cdot \mathrm{spot}^{-1}$ and lidocaine hydrochloride is equal to $1.50 \mu \mathrm{g} \cdot \mathrm{spot}^{-1}$ are demonstrated in Table 3 . The content of both substances in the examined product was determined on the basis of previously described linear equation of the calibration curves determined for hydrocortisone acetate and lidocaine hydrochloride, respectively (Table 2). Moreover, in Table 3, the full statistical analysis of obtained results was performed. Percentage content of hydrocortisone acetate in the examined drugin injection dosage form determined by developed TLC-densitometry is $95.0 \%$ in the relation to the amount declared by manufacturer. This fact shows that obtained hydrocortisone acetate amount complies with the United States Pharmacopoeia specification (monograph) for content of the hydrocortisone acetate in pharmaceutical formulations. According to the United States Pharmacopoeia, the recommended hydrocortisone acetate amount in pharmaceutical formulation administered via injection should be in the range from $90 \%$ to $110 \%$.

Our results presented in Table 3 show that in the case of lidocaine hydrochloride, the content of this substance in the examined combined pharmaceutical preparation in comparison with the value given by manufacturer is $98.4 \%$, that is consistent with that recommended by Polish and United States Pharmacopoeias. The lidocaine hydrochloride content in injection solutions according to both Pharmacopoeias should be in the range from $95 \%$ to $115 \%$ of the declared value.

Summarizing the results obtained in this study, it can be said that the validation process of an analytical method including the TLC-densitometric method developed in this work is a very powerful tool, necessary in quantitative determination of hydrocortisone acetate amount not only in simple pharmaceutical formulations but in combined dosage forms like, for example, injection solution too $(5 \mathrm{~mL}$ ampoule). Because the validation report indicates that the developed method fulfills the validation criteria of an analytical method designated for quantity control of pharmaceutical formulations in terms of specificity, linearity, limit of detection, limit of quantification, precision, accuracy, and robustness, it can be suggested that the TLC-densitometric method, mentioned in this study, is suitable for the routine analysis of hydrocortisone acetate and lidocaine hydrochloride in quantity control laboratories. Moreover, the results of our TLC-method are comparable with those obtained by the use of RP-HPLC method [16]. Thus, it can be concluded that this method can be used as a substitute method for the accurate assay of the hydrocortisone acetate and lidocaine hydrochloride in combined formulations in form of injection solutions, for example, in situation when HPLC or GC is not affordable in laboratory.

\section{Conclusions}

The work highlights that an important advantage of developed TLC-densitometric method is that it can be successfully applied to determination of individual components of analyzed preparation such as hydrocortisone acetate and lidocaine hydrochloride, respectively, in combined dosage forms. The chromatographic plates precoated with silica gel $60 \mathrm{~F}_{254}$ and a mixture of chloroform + acetone + ammonia (25\%) in volume composition $8: 2: 0.1$ as mobile phase resulted in compact bands and symmetric peaks of lidocaine hydrochloride and also hydrocortisone acetate. UV densitometry was performed in absorbance mode at $200 \mathrm{~nm}$ and $250 \mathrm{~nm}$. Under these optimum conditions, complete separation of both bioactive substances and related substances to hydrocortisone acetate, namely, hydrocortisone, prednisolone, and cortisone acetate, are observed. The studies indicated that none of the excipients presented above interfered with the proposed assay method. This aspect of TLC-densitometric analysis is of great interest as it offers possibilities for the assay of hydrocortisone acetate and lidocaine hydrochloride in a combined dosage formulation like injection solution.

The validation performed in accordance with $\mathrm{ICH}$ guidelines and with Ferenczi-Fodor and Nagy-Turák papers of the developed TLC-densitometry in terms of specificity, range, linearity, accuracy, precision, detection limit, quantification limit, and robustness indicates that the elaborated method realizes the criterion of the linearity in required range of hydrocortisone acetate and lidocaine hydrochloride concentrations. The results of hydrocortisone acetate and lidocaine hydrochloride obtained from examined commercial product compared with the value given by manufacturer are consistent with those which are recommended by Polish and United States Pharmacopoeias. It could be said that the NPTLC-densitometric method is a new suitable method for the routine analysis of hydrocortisone acetate and lidocaine hydrochloride in quantity control laboratories.

\section{Conflict of Interests}

The authors have no conflict of interest or no financial gains in mentioning the company names or trademarks.

\section{Acknowledgment}

This research was financed by the Medical University of Silesia as part of statutory research project KNW-1-013/N/3/0.

\section{References}

[1] B. Rocchi and F. Vittorelli, "Determination of chloramphenicol combined with hydrocortisone acetate by ultraviolet spectrophotometry," Bollettino Chimico Farmaceutico, vol. 96, no. 7, pp. 283-286, 1957.

[2] K. Furoda and G. Hashizume, "Quantitative analysis of active components in ointments by x-ray diffractometry. IV. Analysis of hydrocortisone acetate, chloramphenicol, and tetracycline hydrochloride in ointments," Yakugaku Zasshi, vol. 87, no. 5, pp. 500-506, 1967.

[3] D. M. Hailey and A. R. Lea, "High performance liquid chromatographic analysis of hydrocortisone acetate ointments: interlaboratory study," Journal of AOAC International, vol. 64, no. 4, pp. 870-874, 1981. 
[4] A. R. Lea, J. M. Kennedy, and G. K. Low, "Analysis of hydrocortisone acetate ointments and creams by high-performance liquid chromatography," Journal of Chromatography, vol. 198, no. 1, pp. 41-47, 1980

[5] L. Nováková, L. Matysová, and P. Solich, "Advantages of application of UPLC in pharmaceutical analysis," Talanta, vol. 68, no. 3, pp. 908-918, 2006.

[6] M. Amin and U. Jakobs, "Thin-layer chromatography of active compounds from ointments and suppositories followed by direct quantitative analysis by remission," Journal of Chromatography, vol. 131, pp. 391-398, 1977.

[7] Polish Pharmacopoeia, Polish Pharmaceutical Society, Warsaw, Poland, 2011.

[8] The United States Pharmacopoeia, Rockville, Md, USA, 2011.

[9] E. Weijden, M. P. Broek, and F. F. Ververs, "Easy and fast LCMS/MS determination of lidocaine and MEGX in plasma for therapeutic drug monitoring in neonates with seizures," Journal of Chromatography B, vol. 881-882, pp. 111-114, 2012.

[10] L. Chen, L. Liao, Z. Zuo et al., "Simultaneous determination of nikethamide and lidocaine in human blood and cerebrospinal fluid by high performance liquid chromatography," Journal of Pharmaceutical and Biomedical Analysis, vol. 43, no. 5, pp. 17571762, 2007.

[11] Z. Chik, A. Johnston, A. T. Tucker, R. T. Burn, and D. Perrett, "Validation and application of capillary electrophoresis for the analysis of lidocaine in a skin tape stripping study," Biomedical Chromatography, vol. 21, no. 8, pp. 775-779, 2007.

[12] R. T. Coutts, G. A. Torok-Both, Y. K. Tam, L. V. Chu, and F. M. Pasutto, "Acetylation and pentafluorobenzoylation of lidocaine metabolites in aqueous solution and identification of derivatives by combined gas chromography/mass spectrometry," Biomedical and Environmental Mass Spectrometry, vol.1494, pp. 173-182, 1987.

[13] S. Tarek, T. S. Belal, A. Rasha, S. Shaalan, and R. S. Haggag, "Gradient HPLC-diode array detector stability-indicating determination of lidocaine hydrochloride and cetylpyridinium chloride in two combined oral gel dosage forms," Journal of AOAC International, vol. 94, no. 2, pp. 503-512, 2011.

[14] S. Salas, B. Talero, A. M. Rabasco, and M. L. GonzálezRodríguez, "Development and validation of a reverse-phase liquid chromatographic method for the assay of lidocaine hydrochloride in alginate-Gantrez microspheres," Journal of Pharmaceutical and Biomedical Analysis, vol. 47, no. 3, pp. 501507, 2008.

[15] A. H. M. Sarrafi, M. Bahmaei, and A. Z. Mousavi, "Simultaneous spectrophotometric determination of lidocaine and hydrocortisone acetate in pharmaceutical preparations by multivariate calibration methods," Journal of the Iranian Chemical Research, vol. 3, pp. 31-36, 2010.

[16] B. Jancic-Stojanović, A. Malenović, S. Marković, D. Ivanović, and M. Medenica, "Optimization and validation of an RPHPLC method for analysis of hydrocortisone acetate and lidocaine in suppositories," Journal of AOAC International, vol. 93, no. 1, pp. 102-107, 2010.

[17] J. M. Lemus Gallego and J. Perez Arroyo, "Comparison of HPLC and multivariate regression methods for hydrocortisone and lidocaine analysis of pharmaceutical preparations," Analytical and Bioanalytical Chemistry, vol. 374, no. 2, pp. 282-288, 2002.

[18] A. Pyka, D. Nabiałkowska, K. Bober, and M. Dołowy, "Comparison of NP-TLC and RP-TLC with densitometry to quantitative analysis of tocopherol acetate in pharmaceutical preparation,"
Journal of Liquid Chromatography and Related Technologies, vol. 34, no. 20, pp. 2548-2564, 2011.

[19] A. Pyka, M. Babuśka-Roczniak, and P. Bocheńska, "Determination of hydrocortisone in pharmaceutical drug by TLC with densitometric detection in UV," Journal of Liquid Chromatography and Related Technologies, vol. 34, no. 9, pp. 753-769, 2011.

[20] P. Bocheńska and A. Pyka, "Determination of acetylsalicylic acid in pharmaceutical drugs by TLC with densitometric detection in UV," Journal of Liquid Chromatography and Related Technology, vol. 35, no. 17, pp. 1346-1363, 2012.

[21] P. Bocheńska and A. Pyka, "Use of TLC for the quantitative determination of acetylsalicylic acid, caffeine, and ethoxybenzamide in combined tablets," Journal of Liquid Chromatography and Related Technology, vol. 36, no. 17, pp. 2405-2421, 2013.

[22] A. Pyka, E. Wiatr, K. Kwiska, and D. Gurak, "Validation thin layer chromatography for the determination of naproxen in tablets and comparison with a pharmacopeil method," Journal of Liquid Chromatography and Related Technologies, vol. 34, no. 10-11, pp. 829-847, 2011.

[23] A. Pyka, M. Budzisz, and M. Dołowy, "Validation thin layer chromatography for the determination of acetaminophen in tablets and comparison with a pharmacopeial method," BioMed Research International, vol. 2013, Article ID 545703, 10 pages, 2013.

[24] M. Dołowy and A. Niestrój, "Densitometric determination of ursodeoxycholic acid in pharmaceutical formulations in form of tablets and capsules," Journal of Liquid Chromatography and Related Technologies, vol. 33, no. 1, pp. 109-117, 2010.

[25] M. Dołowy, "Investigation of identity of dehydrocholic acid in selected pharmaceutical formulations with the use of NP-TLC method," Annales Universitatis Mariae Curie-Skłodowska, vol. 22, no. 4, pp. 67-73, 2009.

[26] "ICH Harmonised Tripartite Guideline: Validation of analytical procedures: text and Methodology," Q2(R1) Geneva, Switzerland, 2005, http://www.ich.org.

[27] K. Ferenczi-Fodor, B. Renger, and Z. Végh, "The frustrated reviewer-recurrent failures in manuscripts describing validation of quantitative TLC/HPTLC procedures for analysis of pharmaceuticals," Journal of Planar Chromatography, vol. 23, no. 3, pp. 173-179, 2010.

[28] K. Ferenczi-Fodor, A. Nagy-Turák, and Z. Végh, "Validation and monitoring of quantitative thin layer chromatographic purity tests for bulk drug substances," Journal of Planar Chromatography, vol. 8, no. 5, pp. 349-356, 1995.

[29] A. Nagy-Turák, Z. Végh, and K. Ferenczi-Fodor, "Validation of the quantitative planar chromatographic analysis of drug substances. III. Robustness testing in OPLC, Journal of Planar Chromatography, vol. 8, no. 3, pp. 188-193, 1995.

[30] L. S. Ettre, "Nomenclature for chromatography (IUPAC Recommendations 1993)," Pure and Applied Chemistry, vol. 65, no. 4, pp. 819-872, 1993. 

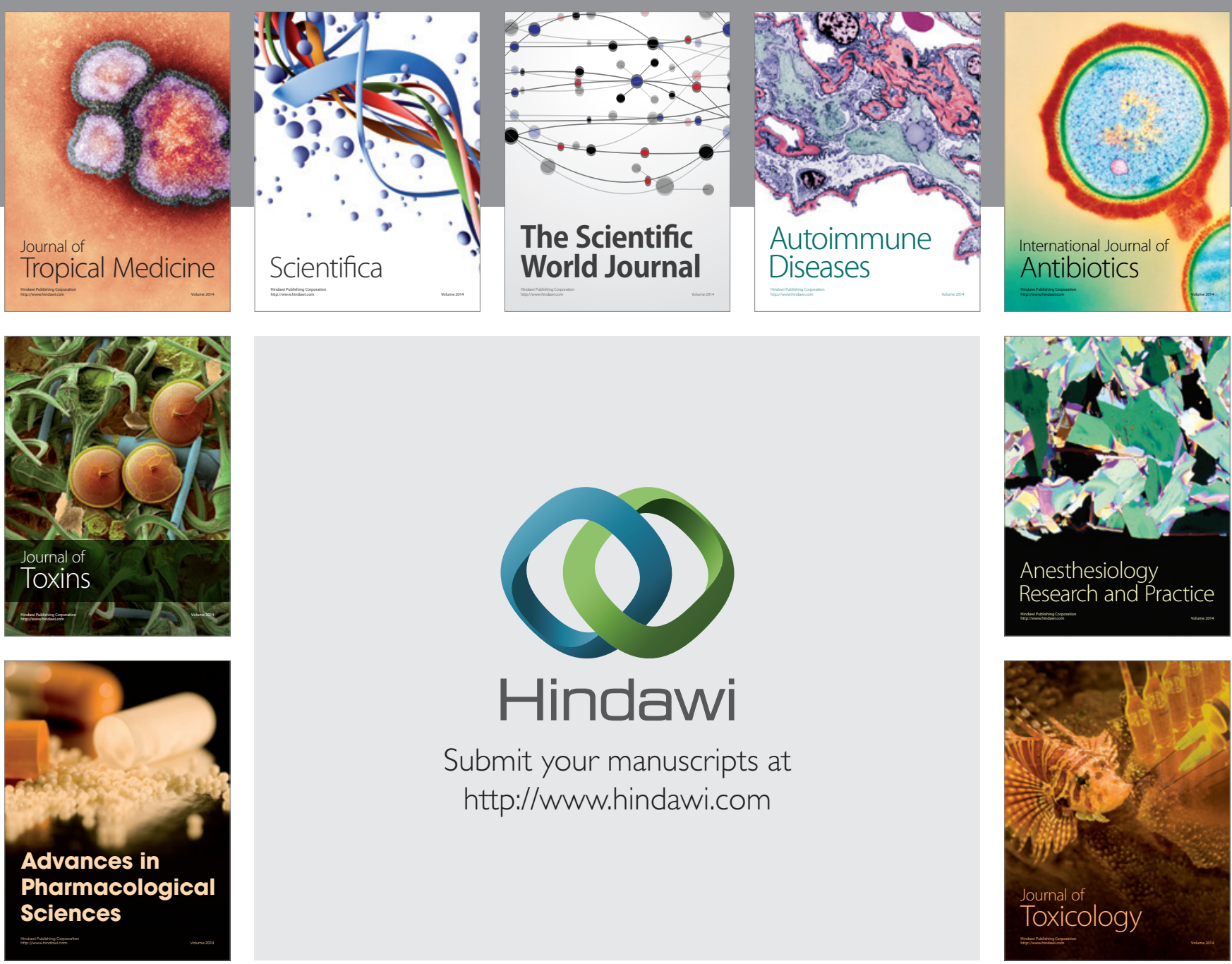

\section{Hindawi}

Submit your manuscripts at

http://www.hindawi.com
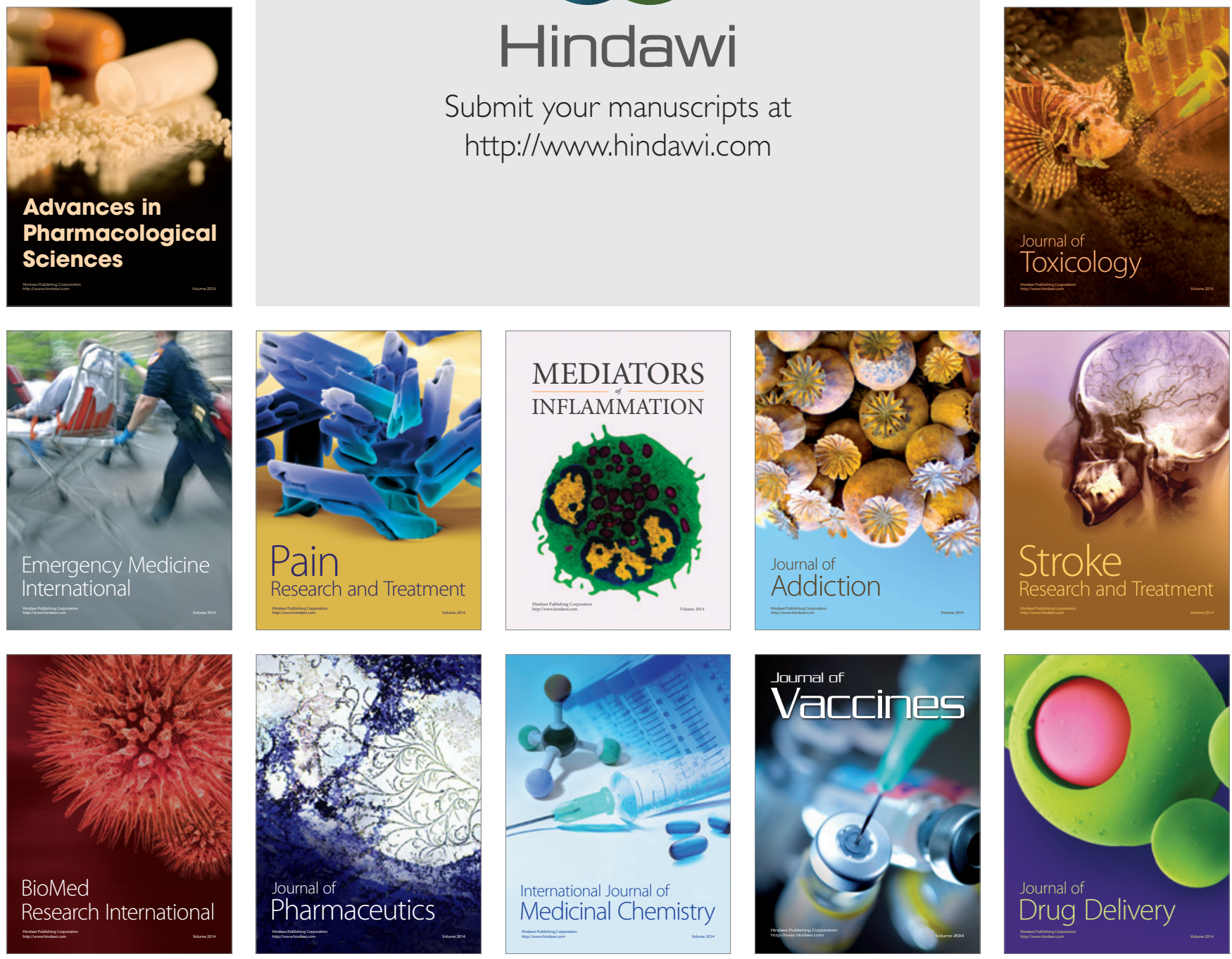